\title{
Trois espèces nouvelles du genRe Medwayella Traub, 1972 (INSECTA : SiPHONAPTERA : PYGIOPSYLLIDAE) de Sabah (Malaisie Orientale, Île de Bornéo)
}

\author{
BEAUCOURNU J.C.* \& WELLS K.**
}

Summary: THREE NEW SPECIES OF MEDWAYELLA (INSECTA: Siphonaptera: Pygiopsyllidae) from Sabah (EAStern Malaysia, BORNEO)

Medwayella traubiana n. sp., M. pfeifferi n. sp. and M. sabahae n. sp. (Pygiopsyllidae) are described from Sabah (north of Borneo island), the first two on Tupaia tana (Scandentia), the last on Sundasciurus lowii (Rodentia). Sex male is only identified, because these fleas have been collected in sympatry, or even in syntopy. Their determination is based on segment IX and aedeagus. If

M. traubiana and $M$. pfeifferi are related to some known species, M. sabahae is clearly distinct from other Medwayella.

KEY WORDS : Sabah, Borneo, Pygiopsyllidae, new species, Medwayella, Medwayella traubiana, Medwayella pfeifferi, Medwayella sabahae.

\section{Résumé :}

Medwayella traubiana n. sp., M. pfeifferi n. sp. et M. sabahae n. sp. (Pygiopsyllidae) sont décrites de Sabah (nord de l'île de Bornéo); les deux premières sur Tupaia tana (Scandentia), la troisième sur Sundasciurus lowii (Rodentia). Seuls les mâles peuvent être séparés, ces puces ayant été récoltées en sympatrie, voire en syntopie. Leur détermination est basée sur le segment IX et l'aedeagus; si les deux premières sont affiliées à des espèces déjà connues, M. sabahae est nettement séparée des autres Medwayella.

MOTS CLÉS: Sabah, Bornéo, Pygiopsyllidae, espèces nouvelles, Medwayella, Medwayella traubiana, Medwayella pfeifferi, Medwayella sabahae.

\section{INTRODUCTION}

A u cours de deux missions scientifiques dans l'île de Bornéo, et précisément dans le territoire de Sabah, l'un de nous (K.W.) a collecté 44 puces appartenant à deux genres et regroupant quatre espèces. Les hôtes sont essentiellement des Tupaiidae (Scandentia), mais aussi quelques Sciuridae (Rodentia).

L'enclave de Sabah (lat. : $5^{\circ} 20 \mathrm{~N}$ - long. : $117^{\circ} 10 \mathrm{E}$ ), appelée autrefois Bornéo septentrional, bien qu'appartenant politiquement à la Malaisie (Malaisie orientale), doit, sur un plan biogéographique, être étudiée comme faisant partie intégrante de l'île de Bornéo dont elle constitue la pointe nord. Ce territoire d'environ $80500 \mathrm{~km}^{2}$ (Bornéo, dans sa totalité en fait 736000 , ce qui la classe comme troisième île du monde pour sa superficie) a un relief tourmenté (le Mont Kinabalu y culmine à $4101 \mathrm{~m}$ ), principalement couvert de forêts, mais, çà et là, des clairières cultivées trouent la couverture arborée.

Les plus importantes récoltes de Puces sur Bornéo (s.l.) furent faites et en partie étudiées par Traub dont le

\footnotetext{
* Laboratoire de Parasitologie appliquée, Faculté de Médecine, 2, avenue du Professeur Léon Bernard, F-35043 Rennes cedex, et Institut de Parasitologie de l'Ouest, même adresse.

** Department of Experimental Ecology (Bio III), University of Ulm, Albert-Einstein Allee 11, D-89069 Ulm.

Correspondance: Jean-Claude Beaucournu.

E-mail : jbeaucou@univ-rennes1.fr
}

travail magistral portant sur les Pygiopsyllidae est de 1972. Cet auteur n'a malheureusement pas eu le temps de terminer la publication du matériel récolté. Durden \& Traub (1990) donnent une liste des genres (et pour chacun, le nombre de taxa) observés, la majorité provenant du Sarawak. Ils notent la présence d'un Ctenophthalmidae (Neopsylla), de 19 Pygiopsyllidae (un Farhangia, deux Lentistivalius, 14 Medwayella et un Gryphopsylla), huit Ceratophyllidae (quatre Macrostylophora et un Paraceras: Ceratophyllinae; deux Cratynius et un Sigmactemus : Leptopsyllinae). Cette liste ne prétendait pas être un catalogue de la faune des Siphonaptères de cet immense berceau insulaire.

Toutes nos captures furent faites en forêts primaires (FP), ou secondaires (FS). 26 spécimens hôtes ont livré des Puces. Nous ne voyons pas d'intérêt à donner un index de fréquence, le but initial de ces missions n'étant pas la collecte des ectoparasites.

Sur les six stations, cinq ont montré des hôtes parasités. Il s'agit, du nord au sud, de :

- Kampung Monggis (FS),

- Kampung Tumbalang (FS),

- Kinabalu Park, Poring Hot Spring (FP),

- Luasong Field Center (FS),

- Tawau Hills National Park (FP).

En tant qu'hôte, Tupaia tana Gray, 1852 est dominant (21 exemplaires parasités), mais aussi dans sa fréquence, suivi de loin par Sundasciurus lowii (Thomas, 1892) (7 ex.), puis par T. longipes (Thomas, 1893) (3 ex.) et Callosciurus prevostii (Desmarest, 1822) (1 ex.). 


\section{SIPHONAPTÈRES COLLECTÉS}

\section{Ceratophyllidae}

- Macrostylophora Ewing, 1929

Un seul mâle figure dans nos collectes. Nous pouvons seulement affirmer qu'il ne s'agit pas de $M$. borneensis (Jordan, 1926), connu cependant du Mont Kinabalu. Le paradoxe est que ce genre compte 19 espèces décrites et une dizaine en attente de description, sans parler des sous-espèces (Traub et al., 1983). Une seule, citée ci-dessus, est nommément connue de Bornéo alors que, nous l'avons dit, quatre sont notées par Durden \& Traub (1990) de cette île.

\section{PygiOPSYLLIDAE}

- Medwayella Traub, 1972

Nous l'avons noté plus haut, Medwayella est le genre le mieux representé à Bornéo, avec 14 taxa, certains pouvant être communs à plusieurs îles (Durden \& Traub, 1990). Les espèces ou sous-espèces qui y sont connues actuellement sont : $M$. robinsoni peregrinata Traub, 1972 (Sarawak occidental), M. phangi tana Traub, 1972 (Sarawak occidental), M. batibacula Traub, 1972 (Sarawak), M. veruta Traub, 1972 (Sabah), M. loncha $($ Jordan, 1926) (Mont Poi = Gunung Poi, Sarawak) et $M$. rhaeba (Jordan, 1926) (Mont Dulit = Gunung Dulit, Sarawak).

M. veruta fut décrite du Mont Kinabalu, zone où nous avons collecté nos Medwayella. Bien que nettement différents, si l'on ne considère que les mâles, il y a donc quatre taxa dans la région de Sabah. Or, nous avons noté dans les stations de Kampung Monggis et de Tawau Hills une sympatrie de deux espèces, attestées par des mâles, différentes dans les deux cas, et une syntopie, deux espèces différentes sur le même hôte, un T. longipes. C'est pourquoi, les femelles étant difficiles, voire impossibles à différencier (Mardon, 1981, dans sa Monographie des Pygiopsyllidae, n'a pas voulu donner de clés des femelles de ce genre), nous ne décrirons pas ce sexe chez nos espèces afin d'éviter des appariements erronés, mais nous précisons que nous avons dans notre matériel, au moins deux, peut-être trois, morphes distincts chez les femelles.

\section{DESCRIPTIONS}

\section{MEDWAYELLA TRAUBIANA N. SP.}

Matériel récolté : mâle holotype sur Tupaia tana Gray, 1852 (Scandentia), Tawau Hills National Park, Sabah, 14 VIII 2003; un mâle paratype sur T. longipes (Thomas, 1893), même station, 8 VIII 2003, en syntopie avec Medwayella n. sp. 3; deux mâles paratypes sur T. tana,
Luasong Field Center, Sabah, 30 VIII 2003; un mâle paratype, même hôte, Kampung Monggis, Sabah, $15 \mathrm{~V}$ 2003; un mâle paratype, même hôte, même station, 16 V 2003; un mâle paratype, même hôte, Kampung Tumbalang, Sabah, 19 VII 2003.

Comme pour les deux nouvelles espèces suivantes, nous ne redonnerons pas les caractères généraux de Medwayella, excellement décrits par Traub (1972), et ne ferons qu'une brève évocation des segments non génitaux. Nous discuterons dans un seul paragraphe la validité des trois espèces décrites ici.

Derivatio nominis : cette espèce est un faible hommage aux travaux de notre regretté collègue et ami, le Professeur Robert Traub qui a recréé, entre autres, la systématique des Pygiopsyllidae.

Dépôt des types : holotype et deux paratypes dans les collections du premier auteur, collections ultérieurement déposées au Muséum national d'histoire naturelle de Paris (Entomologie); les autres paratypes à l'Université d'Ulm.

\section{Description}

Capsule céphalique arrondie, sans soies modifiées; œil grand, pigmenté, réniforme; palpe labial légèrement plus court, ou de même longueur que la coxa I. Thorax : prothorax aussi long que les épines de la cténidie; celles-ci sont au nombre de 20 . Une pseudo-seta nette (éventuellement deux) sous le collier du mesonotum. Segments tarsaux allongés, particulièrement au niveau de la patte III, ce qui est caractéristique d'hôtes arboricoles et sauteurs.

Abdomen (segments non génitaux) : généralement, au moins d'un côté, une spinule sur les tergites II à V, bien que ce dernier en soit quelquefois dépourvu.

Abdomen (segments génitaux) : segment VIII non caractéristique : 30 à 32 soies de taille croissante de l'avant vers l'arrière; marge ventro-distale, entre l'apex et le processus ventral épineux (typique du genre Medwayella), très finement denticulée. Segment IX (figure 1) : basimère classique avec une soie longue sur son lobe ventro-distal et une moyenne à mi-distance de celleci et de l'angulation du lobe dorsal. Telomère avec une stiva ( $c f$. Traub, 1972) nette, mais relativement peu développée, une fois et demie plus longue que haute; bras légèrement sinueux sur son bord ventral, avec au tiers apico-distal, une frange d'environ cinq longues soies pigmentées et plusieurs incolores. Sternite IX : première partie du bras distal très grêle dans sa portion sclérifiée, seule discernable. Bord dorsal présentant deux lobes triangulaires symétriques, l'un aux 3/4 distaux ("subapical lobe" = sal, Traub, 1972; tous les termes anatomiques anglais qui suivront sont empruntés à la terminologie complexe, mais très précise de cet auteur), l'autre formant l'apex ("apical lobe" = al) ; ils sont réunis par une large concavité à fond pratiquement plat, évoquant quelque peu sous cet aspect 
M. limi Traub, 1972. Zone spiculeuse interne ("midmicrospiculate area" $=m m a$ ) en plage. Sur le bord ventral, on note les soies classiques, courtes, larges, incolores, à apex arrondi et quelquefois dilatées ; plus bas, une touffe de longues soies dont les quatre à cinq inférieures sont pigmentées.

Phallosome (figure 4) : lobe dorso-médian de l'aedeagus court dans sa partie apicale qui est doucement arquée par rapport à la partie antérieure; capuchon ("hood" = b) rectiligne, puis sinueux et se terminant par une vaste concavité, jusqu'au rabat ("deltoid flap of hood" $=d f$ ) qui montre un angle net, puis un large lobe régulièrement arrondi. Les deux portions du sclérite de Ford, ou securifer $(=s)$, sont divergentes. Quasicrochet $(=q s)$ allongé, peu large à la base, très étroit à l'apex. Tubus interior $(=t i)$ doucement arqué vers le haut. Phylax $(=p h)$ large, particulièrement dans sa partie ventrale.

Femelles actuellement non reliables à un taxon déjà décrit ou en cours de description dans cet article ( $c f$. paragraphe ci-dessus : genre Medwayella).

Dimensions (insectes mâles, montés) : holotype, 3,0 mm; variations : 2,7 à 3,2 ; moyenne : 2,9 .

\section{MEDWAYELLA PFEIFFERI N. SP.}

Matériel d'étude : mâle holotype sur Tupaia tana, Kinabalu Park, Poring Hot Spring, Sabah, 4 VI 2003; un mâle paratype, même hôte, même station, 11 VI 2003; un mâle paratype, même hôte, Kampung Monggis, Sabah, 23 XI 2002; un mâle paratype, même hôte, même station, 6 V 2003; un mâle paratype, même hôte, même station, $11 \mathrm{~V} 2003$.

Derivatio nominis : cette espèce est nommée en mémoire du Professeur M. Pfeiffer de l'université d'Ulm, qui fut le mentor de l'un d'entre nous (K.W.).

Dépôt des types : comme pour M. traubiana, holotype et un paratype dans les collections du premier auteur, les autres à Ulm.

\section{Description}

Capsule céphalique comme chez $M$. traubiana. Palpe labial un peu plus court que la coxa I.

Thorax : cténidie prothoracique de 20 épines. Pseudoseta présente.

Abdomen (segments non génitaux) : une spinule présente sur les tergites II à V.

Abdomen (segments génitaux) : segment VIII non caractéristique : 25 à 28 soies, sans compter celles du processus épineux; marge ventro-distale, entre l'apex et le processus, lisse. Segment IX (figure 2) : basimère et telomère, comme chez $M$. traubiana. Sternite : bras distal montrant un apex acuminé, non transformé en lobe, réuni à un tubercule émoussé sous-jacent par une marge rectiligne. Zone spiculeuse interne en ligne courbe, de contour variable, figurant plus ou moins un hameçon. Soies émoussées et incolores présentes, au nombre moyen de six sur le bord ventro-apical, suivies à distance vers le bas, de trois soies (en moyenne), longues et pigmentées; entre les deux, trois à cinq soies, plus courtes, non colorées. Partie basale sclérifiée du bras distal plus large que chez l'espèce précédente.

Phallosome (figure 5) : lobe dorso-médian de l'aedeagus relativement long et se prolongeant vers l'avant sans courbure nette. Capuchon continuant la courbe du lobe, puis montrant une petite concavité apicale et une large courbe concave également, jusqu'au rabat; celui-ci est doucement arrondi à son sommet, puis forme une vaste courbe. Les deux bras du securifer sont très divergents, et l'inférieur montre un apex recourbé vers le haut. Quasi-crochet à sommet semblant dédoublé, plus étroit que sa base. Tubus interior sinueux, son méat $(=m)$ tourné vers le haut. Phylax carré à sa base, celle-ci étant relativement étroite.

Femelles : même cas que pour $M$. traubiana.

Dimensions (insectes mâles, montés) : holotype, 3,4 mm; variation : 3,0 à 3,6; moyenne : 3,2.

\section{MEDWAYELLA SABAHAE N. SP.}

Matériel de description : mâle holotype sur Sundasciurus lowii (Thomas, 1892), Tawau Hills National Park, Sabah, 9 VIII 2003; un mâle paratype sur le même hôte, même station, 9 VIII 2003; un mâle paratype sur le même hôte, même station, 18 VIII 2003; un mâle paratype sur Tupaia longipes (Thomas, 1893), même station, 11 VIII 2003, en syntopie avec Medwayella traubiana.

Derivatio nominis : nommée en raison de son pays de découverte, le Sabah.

Dépôt des types : comme pour M. traubiana, holotype et un paratype dans les collections du premier auteur, les autres paratypes à Ulm.

\section{Description}

Capsule céphalique : comme chez M. traubiana; palpe labial atteignant, ou presque, l'apex de la coxa I.

Thorax : cténidie de 18 épines. Pseudo-seta présente sous le collier du mesonotum. Même allongement des segments tarsaux que chez les espèces précédentes. Abdomen (segments non génitaux) : une spinule sur les tergites II à V.

Abdomen (segments génitaux) : segment VIII, non caractéristique : 32 soies sur sa face externe (sans compter celles du processus épineux); marge ventrodistale lisse entre l'apex et le processus épineux. Segment IX (figure 3) : basimère à apex dorsal acuminé; deux soies sur son lobe ventral, soies de tailles très différentes, la plus antérieure faisant, à peu près le tiers de la postérieure qui est effectivement très longue. 

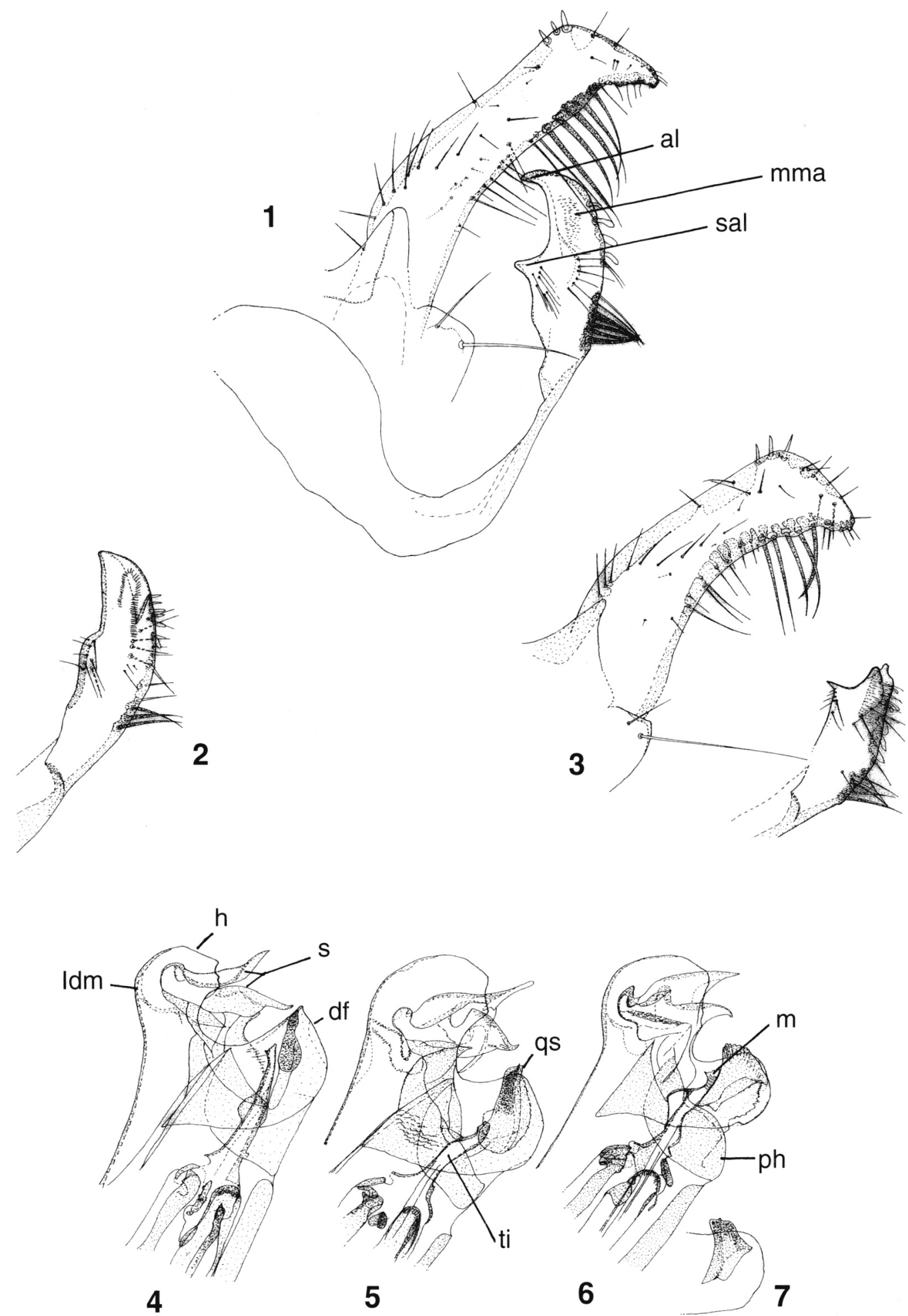

Fig. 1. - Medwayella traubiana n. sp., segment IX (partim) de l'holotype; al : apical lobe; mma : mid-microspiculate area; sal : subapical lobe.

Fig. 2. - Medwayella pfeifferi n. sp., bras distal du sternite IX de l'holotype.

Fig. 3. - Medwayella sabahae n. sp., segment IX (partim) de l'holotype.

Fig. 4. - M. traubiana, apex de l'aedeagus d'un paratype (sur Tupaia tana, Luasong Field Center); $b$ : hood; ldm : lobe dorso-median; $s$ : securifer; $d f$ : deltoid flap.

Fig. 5. - M. pfeifferi, apex de l'aedeagus d'un paratype (sur Tupaia tana, Kampung Monggis); qs : quasi-crochet; $t i$ : tubus interior. Fig. 6 - M. sabahae, apex de l'aedeagus d'un paratype (sur Sundasciurus lowii, Tawau Hills National Park); $m: m e ́ a t ; p h: p h y l a x$. Fig. 7. - M. sabahae, quasi-crochet $(q s)$ et rabat $(d f)$ de l'holotype. 
Telomère plus large et plus court que chez les deux espèces précédentes, avec une stiva courte, pas plus longue que haute; cinq soies pigmentées sur sa marge ventrale. Sternite original à l'intérieur du genre Medwayella : partie apicale dédoublée, avec donc deux apex, le plus externe en lobe triangulaire, le plus interne, un peu plus saillant, à pointe obtuse. Le premier est réuni, sur la marge dorsale, à un lobe pointu, saillant, par une courbe concave très régulière. Zone spiculeuse rectangulaire. Sur le bord ventral, on note six soies incolores, larges, courtes, à apex arrondi pour les quatre supérieures; elles se répartissent en deux séries : les trois supérieures, petites, puis deux ou trois soies classiques et la série inférieure composée de trois soies massives. Plus bas, trois soies longues et pigmentées. Dans son ensemble, la partie apicale du sternite est plus quadrangulaire que triangulaire comme elle se présente chez les autres Medwayella.

Phallosome (figures 6 et 7) : lobe dorso-médian régulièrement arrondi dans sa partie apicale; il montre une nette flexion par rapport à la partie antérieure. Capuchon en continuité avec la courbure du lobe, puis sinueux et aboutissant au rabat en lobe arrondi. Les deux bras du securifer sont peu divergents et de conformation assez voisine : lobes en triangles isocèles, l'inférieur à apex courbé vers le bas. Quasi-crochet de forme un peu variable, mais toujours strié verticalement, ceci pouvant aboutir à un bord apical crénelé (comparer les figures 6 et 7). Tubus interior rectiligne, bien que son bord ventral soit nettement sinueux; méat évasé, finement strié apicalement, courbé vers le haut. Phylax de forme ovoïde.

Femelles : même cas que pour $M$. traubiana.

Dimensions (insectes mâles, montés) : holotype, $2,7 \mathrm{~mm}$; variation : 2,3 à 2,8 ; moyenne 2,65 .

\section{DISCUSSION}

T est évident que nos trois espèces se scindent en deux "groupes" : M. traubiana et $M$. pfeifferi d'un 1 côté, M. sababae de l'autre : ceci est particulièrement évident à l'examen des sternites IX. Nous les discuterons donc séparément.

Les deux premières s'excluent d'emblée du complexe présentant une nette (et étroite) encoche apicale à l'extrémité dorsale du sternite IX, ex "groupe robinsoni". Elles se séparent de toutes les autres par l'association de la forme du sternite IX et de celle du phallosome. Les taxa les plus proches seraient $M$. phangi phangi Traub, 1972, M. veruta et M. limi Traub, 1972; seule $M$. veruta est connue du Sabah, mais les critères de morphologie sont, en l'occurrence, plus importants que ceux des répartitions connues, et la confusion entre ces espèces et les deux premières ici décrites, ne nous semble pas possible.

M. sabahae est d'une telle originalité de par son sternite IX qu'il est impossible de la confondre avec aucune autre espèce de ce genre.

\section{REMERCIEMENTS}

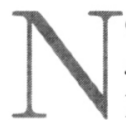
ous remercions l'Economic Planning Unit Malaysia pour nous avoir accordé un Permis de Recherche à Sabah. Nous sommes également reconnaissants aux Sabah Parks, aussi bien qu'au Yayasan Sabah pour leurs autorisations de travailler dans plusieurs zones forestières et pour leurs diverses aides bienveillantes sur le terrain. Le support financier, pendant notre séjour à Sabah, fut aimablement apporté par le Service Allemand Académique d'Échanges (DAAD).

\section{RÉFÉRENCES}

Durden L.A. \& Traub R. Zoogeographical implications from rodent ectoparasites in Sulawesi (pp. 51-62), in: Insects and the rain forests of South East Asia, Wallacea Knight W.J. \& Holloway J.D. (eds). The Royal Entomological Society of London, 1990, IV, 343 p.

MARDON D.K. An illustrated catalogue of the Rothschild collection of Fleas, Vol. VI, Pygiopsyllidae. British Museum (Natural History), 1981, 298 p.

Traub R. The Gunong Benom Expedition, 1967 : 11. Notes on zoogeography, convergent evolution and taxonomy of Fleas (Siphonaptera), based on collections from Gunong Benom and elsewhere in South-East Asia. I. New taxa (Pygiopsyllidae, Pygiopsyllinae). Bulletin of the British Museum (Natural History), Zoology, 1972, 23, 203-305 + $58 \mathrm{pl}$.

Traub R., Rothschild M. \& Haddow J.F. The Rothschild collection of Fleas. The Ceratophyllidae: key to the genera and host relationships. Rothschild M. \& Traub R. (eds), 1983, 288 p. (dont cinq appendices et un index), 205 fig., 90 pl., 152 cartes.

Reçu le 28 avril 2004 Accepté le 28 mai 2004 